gemisch vorhandene neutrale Natriumfluorid. Versuche mit bekannten Gemischen aus reinem $\mathrm{NaF}, \mathrm{NaF}, \mathrm{HF}$ und $\mathrm{Na}_{2} \mathrm{SiF}_{6}$ bestätigen die Brauchbarkeit des Verfahrens.

\title{
Die maßanalytische Bestimmung der höheren Oxyde des Bleis
} and Mangans mit Titantrichlorid wird von L. Moser ${ }^{1}$ ) empfohlen. Der Verfasser gibt einen kurzen Überblick über die verschiedenen Methoden zur Bestimmung des aktiven Sauerstoffs in den genannten Superoxyden ${ }^{2}$ ) und weist besonders auf das empfehlenswerte Verfahren von Schlossberg ${ }^{3}$ ) hin, nach welchem die Reduktion der Superoxyde mit verdünnter Wasserstoffsuperoxydlösung bei Gegenwart von Salpetersäure vorgenommen wird. Ein ebenso einfaches und rasch ausführbares Verfahren hat Moser unter Heranziehung des Titantrichlorids als Reduktionsmittel ausgearbeitet. Da das Titantrichlorid stets etwas freie Salzsäure enthält und diese mit Bleisuperoxyd sehr rasch unter Bildung von freiem Chlor reagiert, muss bei der Analyse von Ble is uperoxyd and von Mennige die Konzentration der Wasserstoffionen im Titanterichlorid durch Wasserzusatz herabgedruckt werden. Es wird daher das abgewogene Bleisuperoxyd zunächst mit etwa $100 \mathrm{ccm}$ Wasser aufgeschlemmt, die Suspension einige Minuten gekocht, um den im Wasser gelösten Sauerstoff zu entfernen, und dann im Kohlensäurestrom die Titantrichloridlösung in raschem Strahle zugegeben, wobei der Kolbeninbalt gut durchgeschüttelt wird. Hervorzuheben ist, dass viel Titantrichlorid auf einmal zugegeben werden muss, da sich sonst trotz der Verdünnang Spuren von Chlor bilden können. Nach erfolgter vollständiger Lösung fügt man etwas Kaliumrhodanidlösung hinza und titriert die noçh warme Flüssigkeit mit Ferrichloridlösung zurück. Man kann auch Methylenblau als Indikator benutzen und muss dann die demselben entsprechende Titantrichlorid-Ménge in Abzug bringen. Wegen der Verdünnung mit Wasser ist es vorteilhaft, die Titanlösung nicht zu schwach herzustellen; man verwendet zweckmäßig eine Lossung, von welcher $1 \mathrm{ccm}$ etwa 0,0035 bis $0,0050 g \mathrm{Fe}$ entspricht. Der Titer der Titanlösung wird in derselben Weise bestimmt; man erhitzt $100 \mathrm{~cm}$ Wasser in einem Kolben etwa 5 Minuten zum Sieden, leitet dann sofort Kohlensäure ein und titriert nach Zusatz einer abgemessenen Menge Titantrichlorid und des Indikators mit der Ferrichloridlösung. Die Verfahren mit Titantrichlorid und mit Wasserstoffsuperoxyd ergaben vollständig identische Resultate, während jene der Bunsen'schen Methode etwas niedriger waren.

Bei der Untersuchung technischer höherer Bleioxyde wird stets auf den Eisengehalt der Produkte Rücksicht zu nehmen sein; seine Bestimmung erfolgt durch vollständige Zersetzung des Superoxydes mit Salzsäure, Entfernung des gelösten Chlors durch Kochen der Flüssigkeit

1) Chem.Ztg. 39, 245 (1915). - Vergl. L. M o ser u. F. See lin g, diese Ztschrft, 52, 73 (1913). - 2) Vergl. diese Ztschrft, 46, 308 (1907), 47, 465 (1908), 50, 209 (1911), 52, 358 (1913) und 53, 261 (1914). - 3) Vergl. diese Ztschrft. 41, 735 (1902). 
in einem schwachen Kohlensäurestrom und Titration des $\mathrm{Fe}^{\cdots}$ - Ions mit Titantrichlorid. Die dem Eisen entsprechende Menge Titanlösung ist dann bei der Bestimmung des aktiven Sauerstoffs in Abzug zu bringen. Ebenso müssen etwa vorhandene Chlorate beachtet und vorher unschädlich gemacht werden. Sie können durch Kochen des Bleisuperoxyds mit etwas Zinkpulver und Essigsäure zu Chloriden reduziert werden, wobei nur zu bedenken ist, dass vor dem Zusatz des Titantrichlorids alles Zink in Lösung gegangen sein muss, weil andernfalls eine partielle Reduktion des gebildeten $\mathrm{Ti}^{\cdots}{ }^{\prime}$-Ions zur $\mathrm{Ti}{ }^{\cdots}$-Stufe stattfinden könnte. Sollte Mangansuperoxyd in der Probe enthalten sein, so würde auch dessen aktiver Sanerstoff wie bei den anderen Methoden mitbestimmt werden.

Bei der Analyse des M a gansuperoxyds macht desseu Schwerlöslichkeit insofern eine Änderung der Arbeitsweise nötig, als die Reduktion in stark salzsaurer Lösung unter Erhitzen vorgenommen werden muss. Daher lässt sich auch Rhodankalium nicht als Indikator verwenden und muss durch Methylenblau ersetzt werden. Am besten verfährt man in folgender Weise: Der feinst gepulverte Braunstein $(0,1$ bis $0,3 g)$ wird anter Einleiten von Kohlensäure mit Titantrichlorid in grossem Überschuss versetzt. Inzwischen wird etwa das gleiche Volumen Salzsäure von 1,19 D. (40 bis $50 \mathrm{ccm}$ ) zur Entfernung der gelösten Laft gekocht, dann in raschem Strahle der Titanlösung hinzugefügt und die salzsaure Lösung unter Umschütteln so lange im schwachen Sieden erhalten, bis sämtliches Mangansuperoxyd in Lösung gegángen ist. Bei natürlichen Braunsteinsorten ist hierzu $1 / 4$ bis $1 / 2$ Stunde und auch länger notwendig. Nach Zusatz ron 4 bis 5 Tropfen Methylenblaulösung wird der Überschuss des Titantrichlorids mit Ferrisalzlösung bestimmt, wobei als Endreaktion der Punlkt gilt, bei welchem die farblose Lösung einen schwach gelbgrünen Ton annimmt. Die Titerstellung der Titanlösung mit dem Ferrichlorid wird in derselben Weise vorgenommen. Der Eisengehalt des Minerals ist auch hier, wie oben angegeben, zu bestimmen und zu berücksichtigeu.

Die beschriebene Methode und das Destillationsverfahren von Bunsen lieferten bei der Untersuchung des Braunsteins gut übereinstimmendeWerte.

Zur Zinkbestimmung in Erzen und Kiesabbränden benutzt H. Rubricius ${ }^{1}$ ) das folgende Verfahren. Es ist besonders für solche Materialien geeignet, welche Zink in geringer Menge enthalten. Man erhitzt $5 \mathrm{~g}$ Substanz mit $15 \mathrm{ccm}$ konzentrierter Salzsäure, gibt zuletzt etwas konzentrierte Șalpetersäure hinzu und engt die Lösung bëi mäßiger Kochhitze bis auf einen kleinen Rest ein, der jedoch noch flüssig bleiben muss, $\mathrm{Zu}$ dem erkalteten Flüssigkeitsrest gibt man etwa $30 \mathrm{ccm}$ konzentriertes Ammoniak und verrührt gut mit einem Glasstabe mit einem Kautschukendchen. Infolge des starken Einengens der I,ösung scheidet sich nur eine ganz geringe Menge eines körnigen Eisenniederschlags ab. Bei der Behandlung mit Ammoniak, die 2- bis 3-mal wiederholt.

1) Chem. Ztg. 39, 198 (1915). 